\title{
First-Order Quantum Phase Transition in a Finite System
}

\author{
A. Leviatan \\ Racah Institute of Physics, The Hebrew University, Jerusalem 91904, Israel
}

(Dated: September 26, 2018)

\begin{abstract}
The dynamics at the critical-point of a general first-order quantum phase transition in a finite system is examined, from an algebraic perspective. Suitable Hamiltonians are constructed whose spectra exhibit coexistence of states corresponding to two degenerate minima in the energy surface separated by an arbitrary barrier. Explicit expressions are derived for wave functions and observables at the critical-point.
\end{abstract}

PACS numbers: 21.60.Fw, 21.10.Re

Quantum phase transitions have become a topic of great interest in different branches of physics [1, 2, 3]. These are structural changes occurring at zero temperature as a function of a coupling constant and driven by quantum fluctuations. The phase transitions are said to be of second-order when the system changes continuously from one phase to another, and of first-order when there is a discontinuous change between two coexisting phases. Advanced experiments have identified such structural changes in a variety of mesoscopic systems, e.g., nuclei, molecules and atomic clusters, where the transitions are between different shapes or geometric configurations. An important issue in these systems is to understand the modifications at criticality due to their finite number of constituents. In the present work we study this question in connection with nuclei exemplifying a finite system undergoing a general first-order shape-phase transition.

The role of a finite number of nucleons can be addressed in the interacting boson model (IBM) [4], which describes low-lying quadrupole collective states in nuclei in terms of a system of $N$ monopole $(s)$ and quadrupole $(d)$ bosons representing valence nucleon pairs. The model is based on a $\mathrm{U}(6)$ spectrum generating algebra and its three solvable dynamical symmetry limits: $U(5), S U(3)$, and $O(6)$, describe the dynamics of stable nuclear shapes: spherical, axially-deformed, and $\gamma$-unstable deformed. A geometric visualization of the model is obtained by an energy surface defined by the expectation value of the Hamiltonian in the coherent (intrinsic) state [5, $\underline{6}$ ]

$$
|\beta, \gamma ; N\rangle=(N !)^{-1 / 2}\left(b_{c}^{\dagger}\right)^{N}|0\rangle,
$$

where $b_{c}^{\dagger}=\left(1+\beta^{2}\right)^{-1 / 2}\left[\beta \cos \gamma d_{0}^{\dagger}+\beta \sin \gamma\left(d_{2}^{\dagger}+d_{-2}^{\dagger}\right) / \sqrt{2}+\right.$ $s^{\dagger}$. For the general IBM Hamiltonian with one- and twobody interactions, the energy surface takes the form

$$
E(\beta, \gamma)=E_{0}+N(N-1) \frac{\left[a \beta^{2}-b \beta^{3} \cos 3 \gamma+c \beta^{4}\right]}{\left(1+\beta^{2}\right)^{2}} .
$$

The coefficients $E_{0}, a, b, c$ involve particular linear combinations of the Hamiltonian's parameters [7, 8]. The quadrupole shape parameters in the intrinsic state characterize the associated equilibrium shape. For $\beta>0$ the intrinsic state is deformed and represents, in a variational sense [9], a ground band whose rotational members are obtained by standard angular momentum projection. Particularly relevant for the present work are states of good $O(3)$ symmetry $L$ projected from the prolatedeformed intrinsic state $|\beta, \gamma=0 ; N\rangle$ of Eq. (1),

$$
\begin{aligned}
|\beta ; N, L, M\rangle & \propto\left[\Gamma_{N}^{(L)}(\beta)\right]^{-1 / 2} \hat{\mathcal{P}}_{L M}|\beta, \gamma=0 ; N\rangle \\
\Gamma_{N}^{(L)}(\beta) & =\frac{1}{N !} \int_{0}^{1} d x\left[1+\beta^{2} P_{2}(x)\right]^{N} P_{L}(x) .
\end{aligned}
$$

Here $P_{L}(x)$ is a Legendre polynomial with $L$ even $(0 \leq$ $L \leq 2 N)$ and $\Gamma_{N}^{(L)}(\beta)$ is a normalization factor. The projected states with fixed $N$ and $L$, involve a mixture of components $\left|N, n_{d}, \tau, L\right\rangle$ with quantum numbers related to the $U(6) \supset U(5) \supset O(5) \supset O(3)$ chain. In general the $L$-projected states $|\beta ; N, L\rangle$ interpolate between the $U(5)$ spherical ground state, $\left|s^{N}\right\rangle \equiv\left|N, n_{d}=\tau=L=0\right\rangle$, at $\beta=0$, and the prolate-deformed ground band with $S U(3)$ character $(\lambda, \mu)=(2 N, 0)$, at $\beta=\sqrt{2}$.

Shape-phase transitions in nuclei have been studied in the geometric framework of a Bohr Hamiltonian for macroscopic quadrupole shapes. Analytic solutions, based on infinite square-well potentials, called $\mathrm{E}(5)$ [1] and $\mathrm{X}(5)$ 2], have been shown to be relevant to the dynamics at the critical-point of second- and firstorder transitions respectively, and empirical examples have been presented [10]. Phase transitions for finite $\mathrm{N}$ can be studied by an IBM Hamiltonian of the form, $H_{1}+g H_{2}$, involving terms from different dynamical symmetry chains [6]. The nature of the phase transition is governed by the topology of the corresponding energy surface (2), which serves as a Landau's potential. IBM Hamiltonians of this type have been studied extensively [11, 12, 13, 14, 15, 16, 17, 18, 19], revealing the finite- $\mathrm{N}$ attributes of the phase transitions. The critical $U(5)-O(6)$ Hamiltonian was found to exhibit a $\gamma$ independent flat-bottomed energy surface, characteristic of a second-order phase transition. The critical $U(5)$ $S U(3)$ Hamiltonian displays an energy surface with degenerate minima separated by an extremely low barrier, hence corresponds to a very specific (and non-generic) first-order phase transition. It is clearly desirable to explore, from an algebraic perspective, the finite-N properties of a general first-order critical-point with an arbitrary barrier. This is the goal of the present investigation. 


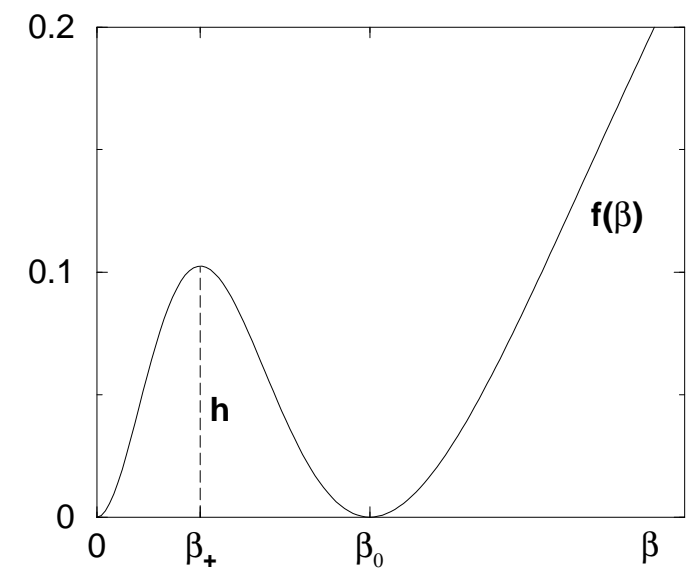

FIG. 1: The energy surface, Eq. (4), at the critical-point of a first-order phase transition. The position and height of the barrier are $\beta_{+}=\left(-1+\sqrt{1+\beta_{0}^{2}}\right) / \beta_{0}$ and $h=f\left(\beta_{+}\right)=$ $\left(-1+\sqrt{1+\beta_{0}^{2}}\right)^{2} / 4$, with $f(0)=f\left(\beta_{0}\right)=0, f(\infty)=1$.

In a first-order phase transition the Landau's potential has two coexisting minima which become degenerate at the critical-point. Such a structure involving spherical and prolate-deformed minima can be accommodated in the energy surface of Eq. (2) provided $a, b>0$ and $b^{2}=$ $4 a c$ at the critical-point. For $\gamma=0$ the corresponding critical energy surface can be transcribed in the form

$$
\begin{aligned}
E_{c r i}(\beta, \gamma=0) & =E_{0}+c N(N-1) f(\beta) \\
f(\beta) & =\beta^{2}\left(1+\beta^{2}\right)^{-2}\left(\beta-\beta_{0}\right)^{2} .
\end{aligned}
$$

$E_{\text {cri }}(\beta, \gamma=0)$, shown in Fig. 1 , behaves quadratically near the minima at $\beta=0$ and $\beta=\beta_{0}=2 a / b>0$, and approaches a constant for large $\beta$. The value of $\beta_{0}$ determines the position $\left(\beta=\beta_{+}\right)$and height $(h)$ of the barrier separating the two minima in a manner given in the caption. $h$ and $\beta_{+}$increase as a function of $\beta_{0}$, and hence both low and high barriers can be considered. To construct a critical Hamiltonian with such an energy surface, it is convenient to resolve it into intrinsic and collective parts [7, 8],

$$
H_{\text {cri }}=H_{\text {int }}+H_{c} .
$$

Such decomposition can be done exactly for any IBM Hamiltonian, as elaborated in great detail in [7, 8]. Adapting this procedure to the critical Hamiltonian, the intrinsic part $\left(H_{\text {int }}\right)$ is defined to have the equilibrium condensate $\left|\beta=\beta_{0}, \gamma=0 ; N\right\rangle$, Eq. (1), as an exact zeroenergy eigenstate and to have an energy surface with a structure as in Eq. (4). $H_{\text {int }}$ is found to have the form

$$
H_{\text {int }}=h_{2} P_{2}^{\dagger}\left(\beta_{0}\right) \cdot \tilde{P}_{2}\left(\beta_{0}\right),
$$

with $P_{2 \mu}^{\dagger}\left(\beta_{0}\right)=\beta_{0} s^{\dagger} d_{\mu}^{\dagger}+\sqrt{7 / 2}\left(d^{\dagger} d^{\dagger}\right)_{\mu}^{(2)}, \tilde{P}_{2 \mu}\left(\beta_{0}\right)=$ $(-1)^{\mu} P_{2,-\mu}\left(\beta_{0}\right)$. Its energy surface coefficients of Eq. (2) are $a=h_{2} \beta_{0}^{2}, b=2 h_{2} \beta_{0}, c=h_{2}, E_{0}=0$, hence satisfy, for $h_{2}>0$, the aforementioned conditions of a first-order critical-point. Since $H_{\text {int }}$ is rotational-scalar, it has, by construction, the $L$-projected states $\left|\beta=\beta_{0} ; N, L\right\rangle$ of Eq. (3) as solvable deformed eigenstates with energy $E=0$. It has also solvable spherical eigenstates: $\left|N, n_{d}=\tau=L=0\right\rangle \equiv\left|s^{N}\right\rangle$ and $\left|N, n_{d}=\tau=L=3\right\rangle$ with energy $E=0$ and $E=3 h_{2}\left[\beta_{0}^{2}(N-3)+5\right]$ respectively. For large $N$ the spectrum of $H_{\text {int }}$ is harmonic, involving quadrupole vibrations about the spherical minimum, and both $\beta$ and $\gamma$ vibrations about the deformed minimum with frequencies $\epsilon, \epsilon_{\beta}$ and $\epsilon_{\gamma}$ given by [8]

$$
\epsilon=\epsilon_{\beta}=h_{2} \beta_{0}^{2} N, \epsilon_{\gamma}=9\left(1+\beta_{0}^{2}\right)^{-1} \epsilon_{\beta} .
$$

For the acceptable range $0 \leq \beta_{0} \leq 1.4$, the $\gamma$-band is expected to be considerably higher than the $\beta$-band. All these features are present in the exact spectrum of $H_{\text {int }}$ shown in Fig. 2, which displays a zero-energy deformed $(K=0)$ ground band, degenerate with a spherical $\left(n_{d}=0\right)$ ground state. The remaining states are either predominantly spherical, or deformed states arranged in several excited $K=0$ bands below the $\gamma$ band. The coexistence of spherical and deformed states is evident in the right portion of Fig. 2, which shows the $n_{d}$ decomposition of wave functions of selected eigenstates of $H_{\text {int }}$. The "deformed" states show a broad $n_{d}$ distribution typical of a deformed rotor structure. The "spherical" states show the characteristic dominance of single $n_{d}$ components that one would expect for a spherical vibrator.

The collective part $\left(H_{c}\right)$ is composed of kinetic terms which do not affect the shape of the energy surface. It can be transcribed in the form [7, 8]

$$
\begin{aligned}
H_{c}= & c_{3}\left[\hat{C}_{O(3)}-6 \hat{n}_{d}\right]+c_{5}\left[\hat{C}_{O(5)}-4 \hat{n}_{d}\right] \\
& +c_{6}\left[\hat{C}_{\overline{O(6)}}-5 \hat{N}\right]+E_{0}
\end{aligned}
$$

where $\hat{N}=\hat{n}_{d}+\hat{n}_{s}, \hat{n}_{d}$ and $\hat{n}_{s}$ are the total-boson, $d$ boson and $s$-boson number operators respectively. Here $\hat{C}_{G}$ denotes the quadratic Casimir operator of the group $\mathrm{G}$ as defined in [8]. In general, the collective and intrinsic parts of the critical Hamiltonian (5D) do not commute. Table I shows the effect of different rotational terms in $H_{c}$. For the high-barrier case considered here, $\left(\beta_{0}=1.3\right.$, $h=0.1$ ), the calculated spectrum resembles a rigid-rotor $\left(E \sim a_{N} L(L+1)\right)$ for the $c_{3}$-term, a rotor with centrifugal stretching $\left(E \sim a_{N} L(L+1)-b_{N}[L(L+1)]^{2}\right)$ for the $c_{5}$-term, and a $\mathrm{X}(5)$-like spectrum for the $c_{6}$-term. In all cases the $\mathrm{B}(\mathrm{E} 2)$ values are close to the rigid-rotor Alaga values. This behaviour is different from that encountered when the barrier is low, e.g., for the critical $U(5)-S U(3)$ Hamiltonian (corresponding to $\beta_{0}=1 / 2 \sqrt{2}$ and $h \approx 10^{-3}$ ), where both the spectrum and E2 rates are similar to the $\mathrm{X}(5)$ predictions [19].

To gain more insight of the underlying structure at the critical-point, we focus the discussion to the lowest coexisting spherical and deformed states. The need for angular momentum projection from mean field solutions in regions of shape-coexistence is well known, e.g., in 

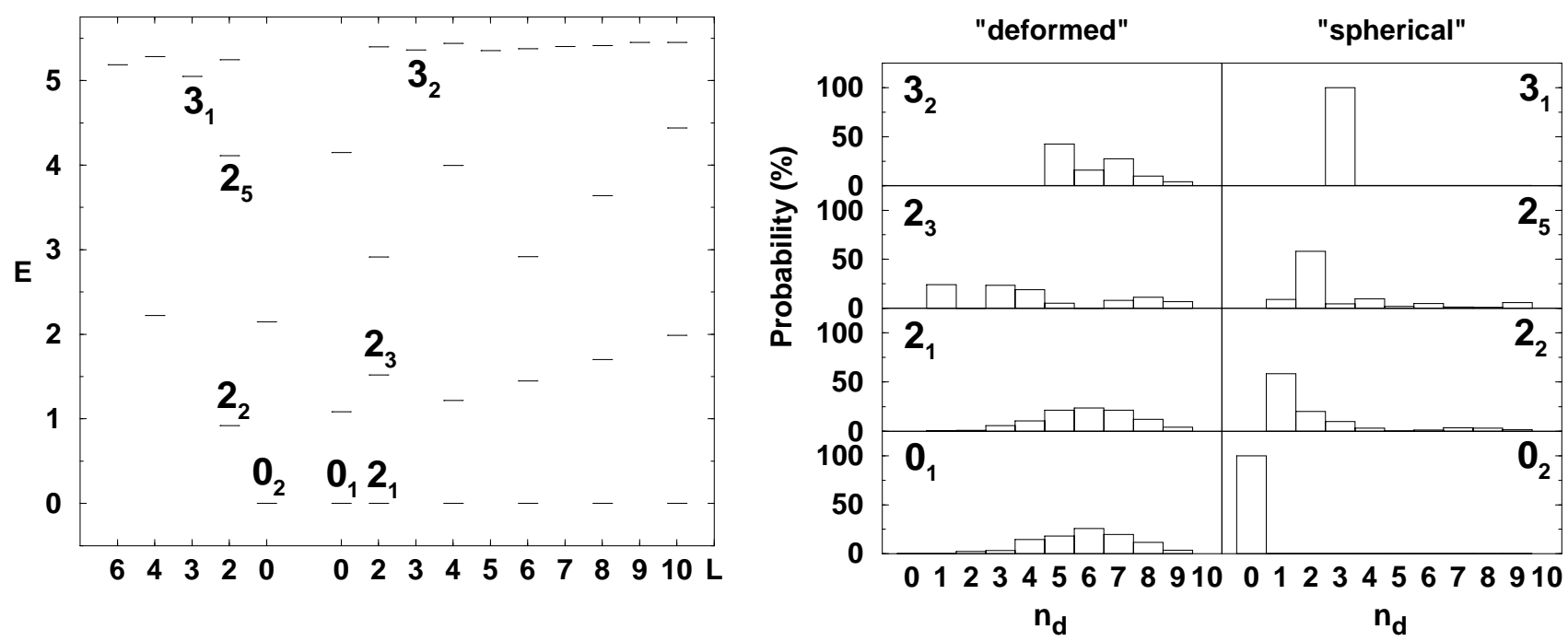

FIG. 2: Left portion: spectrum of $H_{\text {int }}$, Eq. (6), with $h_{2}=0.1, \beta_{0}=1.3$ and $N=10$. Right portion: the number of $d$ bosons $\left(n_{d}\right)$ probability distribution for selected eigenstates of $H_{\text {int }}$.

microscopic calculations employing Hartree-Fock Bogoliubov states [20]. In the present case, the matrix elements of the critical Hamiltonian (5) in the states (3), $E_{L}^{(N)}(\beta)=\left\langle\beta ; N, L\left|H_{c r i}\right| \beta ; N, L\right\rangle=\tilde{E}_{L}^{(N)}(\beta)+E_{0}$, can be evaluated in closed form

$$
\begin{aligned}
\tilde{E}_{L}^{(N)}(\beta)= & h_{2}\left(\beta-\beta_{0}\right)^{2} \Sigma_{2, L}^{(N)}+c_{3}\left[L(L+1)-6 D_{1, L}^{(N)}\right] \\
& +c_{5}\left[D_{2, L}^{(N)}-\beta^{4} S_{2, L}^{(N)}\right] \\
& +c_{6}\left[N(N-1)-\left(1+\beta^{2}\right)^{2} S_{2, L}^{(N)}\right]
\end{aligned}
$$

Here $D_{1, L}^{(N)}, S_{2, L}^{(N)}, D_{2, L}^{(N)}$ and $\Sigma_{2, L}^{(N)}$ denote the expectation values in the states $|\beta ; N, L\rangle$ of $\hat{n}_{d}, \hat{n}_{s}\left(\hat{n}_{s}-1\right)$, $\hat{n}_{d}\left(\hat{n}_{d}-1\right)$ and $\hat{n}_{s} \hat{n}_{d}$ respectively. All these quantities are expressed in terms of the expectation value of $\hat{n}_{s}$, denoted by $S_{1, L}^{(N)}$. Specifically, $D_{1, L}^{(N)}=N-S_{1, L}^{(N)}$, $S_{2, L}^{(N)}=S_{1, L}^{(N)} S_{1, L}^{(N-1)}, \Sigma_{2, L}^{(N)}=(N-1) S_{1, L}^{(N)}-S_{2, L}^{(N)}, D_{2, L}^{(N)}=$ $N(N-1)-2(N-1) S_{1, L}^{(N)}+S_{2, L}^{(N)}$. The quantity $S_{1, L}^{(N)}$ itself is determined by the normalization factors of Eq. (3)

$$
S_{1, L}^{(N)}=\Gamma_{N-1}^{(L)}(\beta) / \Gamma_{N}^{(L)}(\beta) .
$$

It also satisfies the following recursion relation

$$
S_{1, L}^{(N)}=\frac{(N-L / 2)(2 N+L+1)}{\left(\beta^{4}+4\right)(N-1)+3+\left(\beta^{2}-2\right)\left(1+\beta^{2}\right) S_{1, L}^{(N-1)}}
$$

obtained from the fact that $\tilde{P}_{2 \mu}\left(\beta_{0}\right)$ of Eq. (6) annihilates the states $|\beta ; N, L\rangle$ for $\beta_{0}=\beta$. The mixing between the coexisting spherical and deformed $L=0$ states, $\left|\phi_{1}\right\rangle \equiv$ $\left|s^{N}\right\rangle$ and $\left|\phi_{2}\right\rangle \equiv|\beta ; N, L=0\rangle$, can be studied by first transforming to an orthonormal basis

$$
\begin{aligned}
\left|\Psi_{1}\right\rangle & =\left|\phi_{1}\right\rangle,\left|\Psi_{2}\right\rangle=\left(1-r_{12}^{2}\right)^{-1 / 2}\left(\left|\phi_{2}\right\rangle-r_{12}\left|\phi_{1}\right\rangle\right) \\
r_{12} & =\left\langle\phi_{1} \mid \phi_{2}\right\rangle=\left[N ! \Gamma_{N}^{(L=0)}(\beta)\right]^{-1 / 2}
\end{aligned}
$$

and then examining the $2 \times 2$ potential energy matrix,

$$
K_{i j}(\beta)=\left\langle\Psi_{i}\left|H_{c r i}\right| \Psi_{j}\right\rangle=E_{0} \delta_{i j}+\tilde{K}_{i j},
$$

which reads

$$
\begin{aligned}
& \tilde{K}_{11}=0, \tilde{K}_{12}=-c_{6} \beta^{2} \bar{N}\left(1-r_{12}^{2}\right)^{-1 / 2} r_{12}, \\
& \tilde{K}_{22}=\left[\tilde{E}_{L=0}^{(N)}(\beta)+2 c_{6} \beta^{2} \bar{N} r_{12}^{2}\right]\left(1-r_{12}^{2}\right)^{-1},
\end{aligned}
$$

with $\bar{N}=N(N-1)$. The derived eigenvalues of the matrix serve as eigenpotentials, $E_{L=0}^{( \pm)}(\beta)$, and the corresponding eigenvectors, $\left|\Phi_{L=0}^{( \pm)}\right\rangle$, are identified with the ground $\left(0_{1}^{+}\right)$and excited $\left(0_{i}^{+}\right) L=0$ states. The deformed states $|\beta ; N, L\rangle$ of Eq. (3) with $L>0$ are identified with excited members of the ground-band $\left(L_{1}^{+}\right)$ with energies given by $E_{L}^{(N)}(\beta)$, Eq. (9). E2 matrix elements between these states can be evaluated in closed form. For example, for the $E 2$ operator, $T(E 2)=$ $d^{\dagger} s+s^{\dagger} \tilde{d}+\chi\left(d^{\dagger} \tilde{d}\right)^{(2)}$, the necessary matrix elements for transitions involving the $L=0_{1}^{+}, 2_{1}^{+}, 0_{i}^{+}$states are $T_{1} \equiv\left\langle\beta ; N, L^{\prime}=2\|T(E 2)\| \beta ; N, L=0\right\rangle$ and $T_{2} \equiv$ $\left\langle\beta ; N, L^{\prime}=2\|T(E 2)\| s^{N}\right\rangle$,

$$
\begin{aligned}
& T_{1}=\frac{\beta\left[\Gamma_{N-1}^{(0)}(\beta)+(1-\beta \bar{\chi}) \Gamma_{N-1}^{(2)}(\beta)\right]}{\left[\Gamma_{N}^{(2)}(\beta) \Gamma_{N}^{(0)}(\beta)\right]^{1 / 2}}, \\
& T_{2}=\beta N /\left[N ! \Gamma_{N}^{(2)}(\beta)\right]^{1 / 2}
\end{aligned}
$$

with $\bar{\chi}=\sqrt{2 / 7} \chi$. The parameter $\beta$ in the indicated wave functions, energies, and $E 2$ matrix elements, plays the role of an effective deformation whose value is chosen at the global minimum of the lowest eigenvalue, $E_{L=0}^{(-)}(\beta)$, of the matrix $K_{i j}(\beta)$ (13). As is evident from Table I, this procedure leads to accurate finite- $\mathrm{N}$ estimates of observables at the critical-point. The rotational terms 
TABLE I: Excitation energies (in units of $E\left(2_{1}^{+}\right)=1$ ) and $\mathrm{B}(\mathrm{E} 2)$ values [in units of $B\left(E 2 ; 2_{1}^{+} \rightarrow 0_{1}^{+}=1\right)$ ] for selected terms in the critical Hamiltonian, Eqs. (5), (6), (8), with (i) $c_{3} / h_{2}=0.05$, (ii) $c_{5} / h_{2}=0.1$, (iii) $c_{6} / h_{2}=0.05$, and $N=10, \beta_{0}=1.3$, $E 2$ parameter $\chi=-\sqrt{7} / 2$. The entries in square brackets [...] are estimates based on the $L$-projected states, Eq. (3), with (i) $\beta=1.327$, (ii) $\beta=1.318$, (iii) $\beta=1.294$, determined by the global minimum of the respective lowest eigenvalue of the potential matrix, Eqs. (13)-(14). The rigid-rotor and X(5) [2] parameter-free predictions are shown for comparison.

\begin{tabular}{lccccc}
\hline \hline & $c_{3} / h_{2}=0.05$ & $c_{5} / h_{2}=0.1$ & $c_{6} / h_{2}=0.05$ & rotor & $\mathrm{X}(5)$ \\
\hline$E\left(4_{1}^{+}\right)$ & $3.32[3.32]$ & $3.28[3.28]$ & $2.81[2.87]$ & 3.33 & 2.91 \\
$E\left(6_{1}^{+}\right)$ & $6.98[6.97]$ & $6.74[6.76]$ & $5.43[5.63]$ & 7.00 & 5.45 \\
$E\left(8_{1}^{+}\right)$ & $11.95[11.95]$ & $11.23[11.29]$ & $8.66[9.04]$ & 12.00 & 8.51 \\
$E\left(10_{1}^{+}\right)$ & $18.26[18.26]$ & $16.58[16.69]$ & $12.23[12.83]$ & 18.33 & 12.07 \\
$E\left(0_{2}^{+}\right)$ & $6.31[6.30]$ & $6.01[5.93]$ & $4.56[5.03]$ & & 5.67 \\
\hline$B\left(E 2 ; 4_{1}^{+} \rightarrow 2_{1}^{+}\right)$ & $1.40[1.40]$ & $1.40[1.40]$ & $1.46[1.45]$ & 1.43 & 1.58 \\
$B\left(E 2 ; 6_{1}^{+} \rightarrow 4_{1}^{+}\right)$ & $1.48[1.48]$ & $1.48[1.48]$ & $1.55[1.53]$ & 1.57 & 1.98 \\
$B\left(E 2 ; 8_{1}^{+} \rightarrow 6_{1}^{+}\right)$ & $1.45[1.45]$ & $1.45[1.45]$ & $1.53[1.51]$ & 1.65 & 2.27 \\
$B\left(E 2 ; 10_{1}^{+} \rightarrow 8_{1}^{+}\right)$ & $1.37[1.37]$ & $1.37[1.37]$ & $1.44[1.42]$ & 1.69 & 2.61 \\
$B\left(E 2 ; 0_{2}^{+} \rightarrow 2_{1}^{+}\right)$ & $0.003[0.003]$ & $0.003[0.004]$ & $0.24[0.18]$ & & 0.63 \\
\hline \hline
\end{tabular}

in $H_{c}$ (8) affect the value of the effective deformation. This highlights the importance of the coupling between the order-parameter $(\beta)$ and the soft- (rotational) modes at the critical-point of a quantum phase transition [21]. The characteristic spectra, discussed above, of these rotational terms can now be understood from their contribution to $E_{L}^{(N)}(\beta)$, Eq. (9), and $K_{i j}(\beta)$, Eqs. (13)-(14). As observed from Eq. (11), $S_{1, L}^{(N)}$ shows an $L(L+1)$ behaviour near the $S U(3)$ value $\beta=\sqrt{2}$ and, consequently, the contribution of the $c_{3}$-term to $E_{L}^{(N)}(\beta)$ is linear in $L(L+1)$ while that of the $c_{5^{-}}$and $c_{6}$ - terms is parabolic. The rigid- (non-rigid) rotor-like spectrum of the $c_{3^{-}}\left(c_{5^{-}}\right)$ terms then follows from the fact that they contribute only to the diagonal matrix-element $K_{22}(\beta)$ in Eq. (14). The $c_{6}$-term contributes both to diagonal and non-diagonal matrix elements, thus controls the mixing which is essential for obtaining an $X(5)$-like spectrum.

To summarize, we have considered properties of a firstorder quantum shape-phase transition in a finite system from an algebraic perspective. A suitable critical-point Hamiltonian was constructed by resolving it into intrinsic and collective parts. The intrinsic part generates an energy surface with two degenerate minima separated by an arbitrary barrier, and its spectrum exhibits coexistence of spherical and deformed states. The collective part contains kinetic rotational terms which affect the rotational splittings and mixing. Spectral signatures for the case of a high-barrier have been shown to differ from those of a low-barrier. The dynamics at the criticalpoint can be described by an effective deformation determined by variation after projection, combined with two-level mixing of $L=0$ states. Wave functions of a particular analytic form can be used to derive estimates for energies and quadrupole rates at the critical-point. The intrinsic-collective resolution constitute an efficient method for studying shape-phase transitions, since the derived Hamiltonian is tailored to reproduce a given energy surface which, in-turn, governs the nature of the phase transition. Although we have treated explicitly the IBM with one type of bosons, the tools developed are applicable to other finite systems described by similar algebraic models, e.g., the proton-neutron version of the IBM for nuclei [4] and the vibron model for molecules [22]. This work was supported by the Israel Science Foundation.
[1] F. Iachello, Phys. Rev. Lett. 85, 3580 (2000).

[2] F. Iachello, Phys. Rev. Lett. 87, 052502 (2001).

[3] M. Vojta, Rep. Prog. Phys. 66, 2069 (2003).

[4] F. Iachello and A. Arima, The Interacting Boson Model, Cambridge Univ. Press, Cambridge 1987.

[5] J. N. Ginocchio and M. W. Kirson, Phys. Rev. Lett. 44, 1744 (1980).

[6] A. E. L. Dieperink, O. Scholten and F. Iachello, Phys.
Rev. Lett. 44, 1747 (1980).

[7] M.W. Kirson and A. Leviatan, Phys. Rev. Lett. 55, 2846 (1985).

[8] A. Leviatan, Ann. Phys. (NY) 179, 201 (1987).

[9] K. Hagino, G.F. Bertsch, and P.-G. Reinhard, Phys. Rev. C 68, 024306 (2003).

[10] R.F. Casten and N.V. Zamfir, Phys. Rev. Lett. 85, 3584 (2000); 87, 052503 (2001). 
[11] F. Iachello, N.V. Zamfir and R.F. Casten, Phys. Rev. Lett. 81, 1191 (1998).

[12] R.F. Casten, D. Kusnezov and N.V. Zamfir, Phys. Rev. Lett. 82, 5000 (1999).

[13] N.V. Zamfir, P. von Brentano, R.F. Casten and J. Jolie, Phys. Rev. C 66, 021304(R) (2002).

[14] A. Leviatan and J. N. Ginocchio, Phys. Rev. Lett. 90, 212501 (2003).

[15] J.M. Arias, J. Dukelsky and J.E. García-Ramos, Phys. Rev. Lett. 91, 162502 (2003).

[16] F. Iachello and N.V. Zamfir, Phys. Rev. Lett. 92, 212501 (2004).
[17] D.J. Rowe, P.S. Turner and G. Rosensteel, Phys. Rev. Lett. 93, 232502 (2004).

[18] S. Dusuel, J. Vidal, J.M. Arias, J. Dukelsky and J.E. García-Ramos, Phys. Rev. C 72, 011301(R) (2005).

[19] A. Leviatan, Phys. Rev. C 72, 031305(R) (2005).

[20] R. Rodríguez-Guzmán, J.L. Egido and L.M. Robeldo, Phys. Lett. B 474, 15 (2000).

[21] D. Belitz, T.R. Kirkpatrick and T. Vojta, Rev. Mod. Phys. 77, 579 (2005).

[22] F. Iachello and R.D. Levine, Algebraic Theory of Molecules, Oxford Univ. Press, Oxford 1995. 